\title{
Analysis of a delay-induced mathematical model of cancer
}

\author{
Anusmita Das ${ }^{1}$, Kaushik Dehingia ${ }^{1}$, Hemanta Kumar Sarmah ${ }^{1}$, Kamyar Hosseini ${ }^{2 *}$, Khadijeh Sadri $2^{*}$ (D) and
} Soheil Salahshour ${ }^{3}$

\author{
"Correspondence: \\ kamyar_hosseini@yahoo.com; \\ kh.sadri@uma.ac.ir \\ 2Department of Mathematics, Rasht \\ Branch, Islamic Azad University, \\ Rasht, Iran \\ Full list of author information is \\ available at the end of the article
}

\begin{abstract}
In this paper, the dynamical behavior of a mathematical model of cancer including tumor cells, immune cells, and normal cells is investigated when a delay term is induced. Though the model was originally proposed by De Pillis et al. (Math. Comput. Model. 37:1221-1244, 2003), to make the model more realistic, we have added a delay term into the model, and it has incorporated novelty in our present work. The stability of existing equilibrium points in the delay-induced system is studied in detail. Global stability conditions of the tumor-free equilibrium point have been found. It is shown that due to this delay effect, the coexisting equilibrium point may lose its stability through a Hopf bifurcation. The implicit function theorem is applied to characterize a complex function in a neighborhood of delay terms. Additionally, the presence of Hopf bifurcation is demonstrated when the transversality conditions are satisfied. The length of delay for which the solutions preserve the stability of the limit cycle is estimated. Finally, through a series of numerical simulations, the theoretical results are formally examined.
\end{abstract}

Keywords: Mathematical model of cancer; Stability analysis; Global stability analysis; Hopf bifurcation; Implicit function theorem

\section{Introduction}

Cancer is one of the most difficult diseases to treat and is considered one of the leading causes of death. Fighting cancer is important for public health. For this and other economic reasons, extensive research is going on to understand the mechanism involved in the growth of cancer and to predict the impact of this growth on the system [2].

Mathematical modelling is very important in epidemiology because it can provide insight into the key mechanisms that contribute to the spread of disease and suggest control strategies [3]. It is possible to describe a specific disease with mathematical models of differential equations by considering the most important factors which are assumed to be responsible for it and then derive useful information by solving the equations of the model with suitable techniques. Researchers are using mathematical modelling for different diseases like Covid, mumps, rabies, etc., to name a few. Interested readers are referred to the recent works [4-7].

(c) The Author(s) 2022. This article is licensed under a Creative Commons Attribution 4.0 International License, which permits use sharing, adaptation, distribution and reproduction in any medium or format, as long as you give appropriate credit to the original author(s) and the source, provide a link to the Creative Commons licence, and indicate if changes were made. The images or other third party material in this article are included in the article's Creative Commons licence, unless indicated otherwise in a credit line to the material. If material is not included in the article's Creative Commons licence and your intended use is not permitted by statutory regulation or exceeds the permitted use, you will need to obtain permission directly from the copyright holder. To view a copy of this licence, visit http://creativecommons.org/licenses/by/4.0/. 
Mathematical models of tumor growth are widely studied to understand the pathogenesis and predict its future function. One of the most challenging tasks in creating a mathematical model for cancer treatment is calculating biological parameters from data. This task becomes more difficult when the model incorporates many cell types and treatment modalities [8]. Over the past few decades, several experiments and interventions have helped us understand the growth process and its interaction with the immune system.

Many models in the literature represent the dynamics of the systems that vary without time delay [3, 8-11]. To show the realistic nature of the models, it makes sense to incorporate time delays into the systems. Inclusion of delays in mathematical models makes them more realistic by explaining the effects of disease latency or immunity [12-14]. Specifically, the human immune system contains necessary detection systems and weapons to counterattack foreign bodies. This system plays an important role in the prevention of many diseases [15]. When abnormal cells appear in the body, the immune system is activated, and it tries to identify and destroy those abnormal cells. In such a process, the immune system will take some time to provide the right response after discovering the non-self-cells [16-18]. As a result, incorporating the delay term makes the model more realistic. Generally, when the system loses stability due to delays, other powerful nonlinear factors such as oscillatory behavior and bifurcation may occur [19-22]. Accordingly, the analysis of nonlinear dynamics has attracted the attention of many authors in recent years.

Time delay plays an important role in modeling the dynamics of multispecies interactions. Many researchers studied the delay-induced dynamics in predator-prey systems. Beretta and Takeuchi [23] discussed the global stability of the Lotka-Volterra autonomous model with diffusion and delay of time. The delay in the model proposed by Kuznetsov and Taylor was studied by Galach [13], where the delay in time was introduced to achieve better consistency with reality. In 2006, Yafia [24] explored the effect of delays on Kuznetsov's model. Xu and Ma [25] studied the SEIRS epidemic model with a saturation incidence rate and time delay defining the latent period. In 2014, Rihan [26] explored a delay differential model, analyzed it numerically, and demonstrated an effective way of combining chemotherapy with therapeutic immunotherapy. Xu et al. [27] studied a mathematical model with the delay effect on tumor growth due to periodic treatment such that their model was based on reaction-diffusion dynamics and mass conservation law which took into account cell proliferation delays. Yua et al. [28] incorporated delays into the model for activation of the effector cells by helper $T$-cells, and after analyzing the results, it was shown that delayed immune response could play a major role in regulating tumor growth. In [29], Malinzi studied a delayed model to report delays resulting from virus-cell infection and chemotherapy responses; based on the analysis, they proposed three druginjection methods: constant, single bolus, and periodic treatment. Laaroussi et al. [30] presented a mathematical model of oncolytic virotherapy that included a long-term delay representing multiple periods of the lytic cycle.

In the present study, we investigate a mathematical model that provides an important connection between tumor and immune cells. The model is represented by a system of three differential equations and was originally proposed by De Pillis et al. [1]. To make the model more realistic, a time delay is incorporated into the prey (immune cells) specific growth function due to tumor cells. This consideration has incorporated some novelty 
into our present investigation. We have analyzed the effect of delay on different dynamical behaviors shown by the model.

The paper is structured as follows: Sect. 2 describes the proposed model and the basic assumptions. In Sect. 3, we examine the positive invariance and boundedness of the model solutions. In Sect. 4, the existence of equilibrium points for the system is discussed. Section 5 deals with finding the nature of stability of these equilibrium points, along with the occurrence of Hopf bifurcation. In Sect. 6, some simulation results are presented. In Sect. 7, we have our conclusion.

\section{Model formulation}

The model presented in this paper is a modified form of that proposed by De Pillis et al. [1]. The modification is carried out by inducing delay terms in the second term viz. Michaelis-Menten term of the first equation of the system with proper biological justifications. Biochemical reactions involving a single substrate are often assumed to follow Michaelis-Menten kinetics, without regarding the model's underlying assumptions. The change is done to see the impact of delay in the complete system.

The equations representing the system are as follows:

$$
\begin{aligned}
& \frac{d M}{d t}=\mu+\frac{\rho M(t-\tau) H(t-\tau)}{\sigma+H(t-\tau)}-m_{1} M H-d_{1} M, \\
& \frac{d H}{d t}=r_{1} H(1-b H)-m_{2} M H-h_{1} H R, \\
& \frac{d R}{d t}=r_{2} R(1-R)-h_{2} R H,
\end{aligned}
$$

where $M(t), H(t)$, and $R(t)$ are the densities of the immune cells, tumor cells, and normal cells, respectively.

The first equation of system (1) represents the rate of change of immune cells with respect to time. The first term, namely $\mu$, represents the constant source rate of immune cells. Immune cells are recruited by tumor cells through the Michaelis-Menten term $\rho M H /(\sigma+H)$, where $\rho$ is the rate at which the immune cells grow, and $\sigma$ represents the steepness of immune response. To describe the time lag by the immune system for developing a suitable response after recognizing the tumor cells, we need to include the effect of time-delay $\tau$ into the Michaelis-Menten term [31]. Hence, a discrete-time delay is added to the second term of the first equation of system (1) and, as a result, the term becomes $\rho M(t-\tau) H(t-\tau) /(\sigma+H(t-\tau))$. The third term, $-m_{1} M H$, represents the kill rate of immune cells due to interaction with tumor cells, while the fourth term indicates that immune cells die off at a rate of $d_{1}$ per day.

In the second equation of system (1), tumor cells are assumed to grow logistically with an intrinsic growth rate $r_{1}$ and the maximum carrying capacity of $b^{-1}$ in the absence of immune cells and drug therapy. Tumor cells are killed by interaction with immune cells and normal cells as shown by the terms $-m_{2} M H$ and $-h_{1} H R$.

In the third equation of system (1), normal cells are also assumed to grow logistically with an intrinsic growth rate $r_{2}$ and the maximum carrying capacity of one. The second term, $-h_{2} R H$, represents the kill rate of the normal cell due to interaction with tumor cells. Below, we have presented the parameter values which we have used for our investigation.

In the next section, we discuss the boundedness and positive invariance of the solutions of the considered model and related subtopics. 


\section{Boundedness and positive invariance}

By following the standard comparison theory, from the equations of system (1), we get

$$
\frac{d M}{d t}=\mu+\frac{\rho M(t-\tau) H(t-\tau)}{\sigma+H(t-\tau)}-m_{1} M H-d_{1} M \leq \mu-d_{1} M .
$$

Integration of the above leads to

$$
M(t) \leq \frac{\mu}{d_{1}}+M(0) e^{-d_{1} t} \Rightarrow \lim _{t \rightarrow \infty} \sup (M(t)) \leq \frac{\mu}{d_{1}} .
$$

Furthermore,

$$
\frac{d H}{d t}=r_{1} H(1-b H)-m_{2} M H-h_{1} H R \leq r_{1} H(1-b H) .
$$

Proceeding as above, we have

$$
H(t) \leq \frac{1}{b+H(0) e^{-r_{1} t}} \Rightarrow \lim _{t \rightarrow \infty} \sup (T(t)) \leq \frac{1}{b}
$$

and similarly, we find

$$
\begin{aligned}
\frac{d R}{d t}=r_{2} R(1-R)-h_{2} R H \leq r_{2} R(1-R) & \Rightarrow \quad R(t) \leq \frac{1}{1+R(0) e^{-r_{2} t}} \\
& \Rightarrow \lim _{t \rightarrow \infty} \sup (R(t)) \leq 1,
\end{aligned}
$$

with the initial conditions $M(0)>0, H(0)>0$, and $R(0)>0$.

Therefore, the numbers of immune-tumor-normal cells are always bounded. Consequently, we get the bounded set

$$
\Delta=\left\{(M(t), H(t), R(t)) \in R_{+}^{3}: 0 \leq M(t) \leq \frac{\mu}{d_{1}}, 0 \leq H(t) \leq \frac{1}{b}, 0 \leq R(t) \leq 1\right\} .
$$

So, the solutions of system (1) are bounded.

The equations representing system (1) are

$$
\begin{aligned}
& \frac{d M}{d t}=\mu+\frac{\rho M(t-\tau) H(t-\tau)}{\sigma+H(t-\tau)}-m_{1} M H-d_{1} M, \\
& \frac{d H}{d t}=r_{1} H(1-b H)-m_{2} M H-h_{1} H R, \\
& \frac{d R}{d t}=r_{2} R(1-R)-h_{2} R H,
\end{aligned}
$$

subject to the following initial conditions:

$$
\begin{aligned}
& M(\xi)=\psi_{1}(\xi), \quad H(\xi)=\psi_{2}(\xi), \quad R(\xi)=\psi_{3}(\xi), \\
& \psi_{1}(\xi) \geq 0, \quad \psi_{2}(\xi) \geq 0, \quad \psi_{3}(\xi) \geq 0, \quad \xi \in[-\tau, 0], \\
& \psi_{1}(0)>0, \quad \psi_{2}(0)>0, \quad \psi_{3}(0)>0,
\end{aligned}
$$


where

$$
S_{+}=\left\{\left(\psi_{1}(\xi), \psi_{2}(\xi), \psi_{3}(\xi)\right) \in S\left([-\tau, 0], R_{+}^{3}\right)\right\}
$$

The delay differential system (1) can be written in the vector form as

$$
\dot{X}=F(X)
$$

with $X=(M, H, R)^{T} \in R_{+}^{3}$ and

$$
F(X)=\left(\begin{array}{c}
\mu+\frac{\rho M(t-\tau) H(t-\tau)}{\sigma+H(t-\tau)}-m_{1} M H-d_{1} M \\
r_{1} H(1-b H)-m_{2} M H-h_{1} H R \\
r_{2} R(1-R)-h_{2} R H
\end{array}\right)=\left(\begin{array}{c}
F_{1} \\
F_{2} \\
F_{3}
\end{array}\right),
$$

where $F \in C^{\infty}\left(R_{+}^{3}\right)$ is defined in the positive quadrant $R_{+}^{3}$ and represents a mapping $F$ : $S_{+} \rightarrow R_{+}^{3}$. The right-hand side of system (3) is locally Lipchitz, meaning that the derivative is bounded and satisfies

$$
\left.F_{i}(X)\right|_{Y_{i}(t)=0, X \in S_{+}}=F_{i}(0), \quad i=1,2,3 .
$$

According to the second lemma in [32], every solution of system (1) with initial conditions (2), $\psi_{i}(t) \in S_{+}$, say $Y(t)=Y(t ; Y(0))$, for all $t>0$, remains positive throughout the domain $S_{+}, \forall t>0$. Therefore, the solutions of (1) are positively invariant in time $t$.

\section{Existence of equilibrium points}

The equilibrium points of model (1) are found by equating each first derivative to zero. Those are found to be:

(i) $P_{1}\left(M_{1}, 0, R_{1}\right)$, the tumor-free equilibrium point. Here, $M_{1} \geq 0$ and $R_{1} \geq 0$ which are found to be

$$
M_{1}=\frac{\mu}{d_{1}}, \quad R_{1}=1
$$

(ii) $P_{2}\left(M_{2}, H_{2}, R_{2}\right)$, the coexisting equilibrium point. Here, $M_{2} \geq 0, H_{2} \geq 0$, and $R_{2} \geq 0$ and

$$
M_{2}=\frac{\mu}{d_{1}+m_{1} H_{2}-\frac{\rho H_{2}}{\sigma+H_{2}}}, \quad H_{2}=\frac{r_{1}-m_{2} M_{2}-h_{1} R_{2}}{r_{1} b}, \quad R_{2}=\frac{r_{2}-h_{2} H_{2}}{r_{2}} .
$$

Note that $\mathrm{H}_{2}$ can be found by solving the equation

$$
H_{2}=\frac{1}{b}-\frac{m_{2}}{r_{1} b}\left(\frac{\mu\left(\sigma+H_{2}\right)}{\left(d_{1}+m_{1} H_{2}\right)\left(\sigma+H_{2}\right)-\rho H_{2}}\right)-\frac{h_{1}}{r_{1} b}\left(\frac{r_{2}-h_{2} H_{2}}{r_{2}}\right) \text {, }
$$

or $A_{1} H_{2}^{3}+A_{2} H_{2}^{2}+A_{3} H_{2}+A_{4}=0$, where

$$
\begin{aligned}
& A_{1}=m_{1} r_{1} r_{2} b-m_{1} h_{1} h_{2}, \\
& A_{2}=\left(\sigma m_{1}+d_{1}-\rho\right)\left(r_{1} r_{2} b-h_{1} h_{2}\right)-m_{1}\left(r_{1} r_{2}-h_{1} r_{2}\right),
\end{aligned}
$$




$$
\begin{aligned}
& A_{3}=\mu r_{2} m_{2}+\sigma d_{1}\left(r_{1} r_{2} b-h_{1} h_{2}\right)-\left(\sigma m_{1}+d_{1}-\rho\right)\left(r_{1} r_{2}-h_{1} r_{2}\right), \\
& A_{4}=\sigma \mu r_{2} m_{2}-\sigma d_{1}\left(r_{1} r_{2}-h_{1} r_{2}\right) .
\end{aligned}
$$

The equilibrium point $P_{2}$ exists if

$$
\left(d_{1}+m_{1} H_{2}\right)\left(\sigma+H_{2}\right)>\rho H_{2}, \quad H_{2} \geq 0, \quad \text { and } \quad r_{2}>h_{2} H_{2} .
$$

\section{Analysis of the stability of the equilibrium points}

In this section, we are interested in studying the nature of stability of the tumor-free equilibrium and the coexisting equilibrium of the system.

\subsection{Local stability analysis of the tumor-free equilibrium point $P_{1}$}

The Jacobian matrix of system $(1)$ at the equilibrium point $P_{1}\left(\frac{\mu}{d_{1}}, 0,1\right)$ is

$$
J_{P_{1}}=\left(\begin{array}{ccc}
-d_{1} & \frac{\rho M_{1}}{\sigma} e^{-\phi \tau}-m_{1} M_{1} & 0 \\
-m_{2} H_{1} & r_{1}-m_{2} M_{1}-h_{1} R_{1} & 0 \\
0 & -h_{2} R_{1} & r_{2}-2 r_{2} R_{1}
\end{array}\right)
$$

At the equilibrium point $P_{1}\left(\frac{\mu}{d_{1}}, 0,1\right)$, the eigenvalues of the Jacobian matrix $J_{P_{1}}$ are

$$
\phi_{1}=-d_{1}<0, \quad \phi_{2}=r_{1}-\frac{m_{2} \mu}{d_{1}}-h_{1}, \quad \phi_{3}=-r_{2}<0 .
$$

By applying the standard result $[3,8]$, the necessary condition for asymptotic stability of equilibrium point $P_{1}$ is found to be $r_{1}<\frac{m_{2} \mu}{d_{1}}+h_{1}$, and this point will be unstable when $r_{1} \geq \frac{m_{2} \mu}{d_{1}}+h_{1}$.

Thus, all eigenvalues have negative real parts if $r_{1}<\left(m_{2} \mu / d_{1}\right)+h_{1}$ for all $\tau \geq 0$. Accordingly, the equilibrium point $P_{1}$ is locally asymptotically stable.

\subsection{Global stability analysis of the tumor-free equilibrium point $P_{1}$}

In this section, using a Lyapunov function, we show that the tumor-free equilibrium point $P_{1}$ is also globally asymptotically stable when $r_{1}<\left(m_{2} \mu / d_{1}\right)+h_{1}$.

To this end, the work [30] is followed to study the dynamics of system (1) when $\tau \geq 0$. We choose $\psi=\left(\psi_{1}, \psi_{2}, \psi_{3}\right)=(M, H, R)$ defined in the space

$$
S_{+}=\left\{\psi \in S\left([-\tau, 0], R_{+}^{3}\right): M(\xi)=\psi_{1}(\xi), H(\xi)=\psi_{2}(\xi), R(\xi)=\psi_{3}(\xi)\right\}
$$

where $\xi \in[-\tau, 0], \psi_{i}(\xi) \geq 0(i=1,2,3)$, and $\psi_{\mathrm{i}}$ are continuous functions on the interval $[-\tau, 0]$.

Considering a Lyapunov function given as

$$
L(\psi)=\psi_{1}(0)+\psi_{2}(0)+\psi_{3}(0)+\int_{-\tau}^{0} \frac{\rho \psi_{1}(s) \psi_{2}(s)}{\sigma+\psi_{2}(s)} d \mathrm{~s}
$$

we find that

$$
\frac{d L(\psi)}{d t}=\frac{d \psi_{1}(0)}{d t}+\frac{d \psi_{2}(0)}{d t}+\frac{d \psi_{3}(0)}{d t}+\frac{\rho \psi_{1}(0) \psi_{2}(0)}{\sigma+\psi_{2}(0)}-\frac{\rho \psi_{1}(-\tau) \psi_{2}(-\tau)}{\sigma+\psi_{2}(-\tau)}
$$




$$
\begin{aligned}
= & \left(\mu+\frac{\rho \psi_{1}(-\tau) \psi_{2}(-\tau)}{\sigma+\psi_{2}(-\tau)}-m_{1} \psi_{1}(0) \psi_{2}(0)-d_{1} \psi_{1}(0)\right) \\
& +\left(r_{1} \psi_{2}(0)\left(1-b \psi_{2}(0)\right)-m_{2} \psi_{1}(0) \psi_{2}(0)-h_{1} \psi_{2}(0) \psi_{3}(0)\right) \\
& +\left(r_{2} \psi_{3}(0)\left(1-\psi_{3}(0)\right)-h_{2} \psi_{2}(0) \psi_{3}(0)\right) \\
& +\frac{\rho \psi_{1}(0) \psi_{2}(0)}{\sigma+\psi_{2}(0)}-\frac{\rho \psi_{1}(-\tau) \psi_{2}(-\tau)}{\sigma+\psi_{2}(-\tau)} \\
= & \left(\mu-m_{1} \psi_{1}(0) \psi_{2}(0)-d_{1} \psi_{1}(0)\right) \\
& +\left(r_{2} \psi_{1}(0)\left(1-b \psi_{2}(0)\right)-m_{2} \psi_{1}(0) \psi_{2}(0)-h_{1} \psi_{2}(0) \psi_{3}(0)\right) \\
& +\left(r_{2} \psi_{3}(0)\left(1-\psi_{3}(0)\right)-h_{2} \psi_{2}(0) \psi_{3}(0)\right)+\frac{\rho \psi_{1}(0) \psi_{2}(0)}{\sigma+\psi_{2}(0)} \\
= & \left(\mu-d_{1} \psi_{1}(0)\right)+\left(r_{2} \psi_{3}(0)\left(1-\psi_{3}(0)\right)\right) \\
= & \mu-d_{1} \frac{\mu}{d_{1}}+r_{2} .1(1-1)=0 .
\end{aligned}
$$

Since $\frac{d L(\psi)}{d t}=0$, we get $\left\{\psi \in R_{+}^{3}: M(\xi)=\psi_{1}(\xi), H(\xi)=\psi_{2}(\xi)=0, R(\xi)=\psi_{3}(\xi)\right\}=P_{1}$.

Now, the classical LaSalle's invariance principle [30] implies that $P_{1}$ is globally attractive. This confirms the global asymptotically stability of $P_{1}$ when $r_{1}<\left(m_{2} \mu / d_{1}\right)+h_{1}$.

\subsection{Simulation results}

To verify the above analytical results, we carry out the following simulations. In doing so, we have considered the parameter values as given in Table 1.

Case 1: $r_{1}<\frac{m_{2} \mu}{d_{1}}+h_{1}$.

Figure 1 shows that when $\tau \geq 0$ and $r_{1}<\left(m_{2} \mu / d_{1}\right)+h_{1}$, the number of tumor cells decreases to zero (approx.) and the growth of immune and normal cells will be stabilized at the tumor-free equilibrium point $P_{1}$. It further shows that when the delay term increases it takes more time to stabilize towards the equilibrium point $P_{1}$.

From the vector field plot (Fig. 2), it is seen that an arbitrary trajectory (in the basin of attraction) starting with different time delays converges to the tumor-free equilibrium point $P_{1}$ (indicated by the black dot) indicating that it is globally stable for system (1) as long as the condition $r_{1}<\left(m_{2} \mu / d_{1}\right)+h_{1}$ is satisfied.

Figure 2 further shows (as in Fig. 1) that, as time lag is increased, complete elimination of the tumor cells takes more time. Biologically, we know that unless the tumor cells get eradicated, the immune system remains active, and it eventually drops to its origi-

Table 1 Parameter values considered for the model

\begin{tabular}{llll}
\hline Parameters & Meaning & Values & Source \\
\hline$\mu$ & Constant source rate of immune cells & 0.05 & {$[1]$} \\
$d_{1}$ & Natural immune cells death rate & 0.2 & {$[1]$} \\
$r_{1}$ & Intrinsic tumor growth rate & 0.4 (Estimated) & {$[1]$} \\
$r_{2}$ & Growth rate of normal cell & 0.35 & {$[1]$} \\
$1 / b$ & Tumor population carrying capacity & $2 / 3$ & {$[1]$} \\
$m_{1}$ & Immune cells kill rate owing to tumor cells & 0.2 & {$[1]$} \\
$m_{2}$ & Tumor cells kill rate owing to immune cells & 0.3 & {$[1]$} \\
$h_{1}$ & Tumor cells kill rate owing to normal cells & 0.2 & {$[1]$} \\
$h_{2}$ & Normal cells kill rate owing to tumor cells & 0.25 & {$[1]$} \\
$\rho$ & Maximum recruitment of immune cells by tumor cells & 1 & {$[1]$} \\
$\sigma$ & Constant half saturation for the proliferation term & 0.4 & {$[1]$} \\
\hline
\end{tabular}




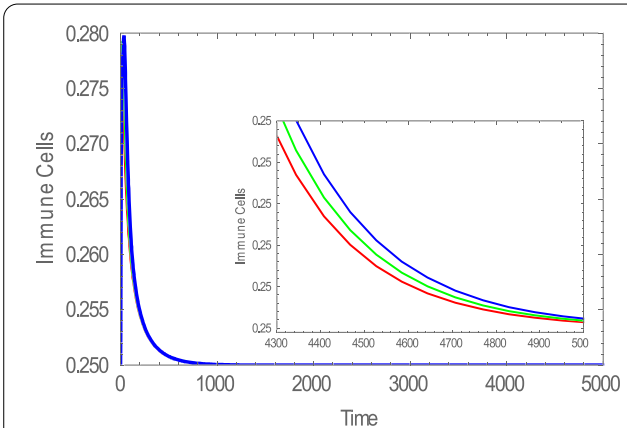

(a)

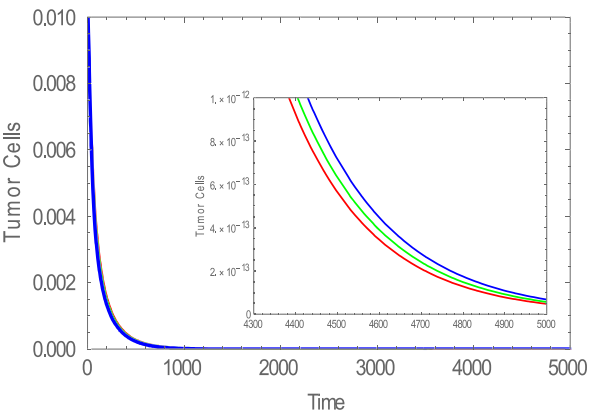

(b)

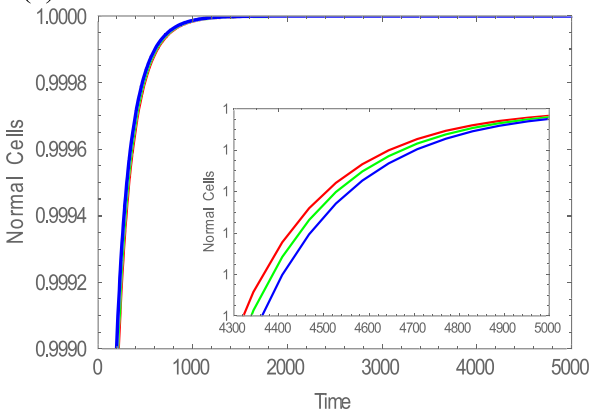

(c)

Figure 1 Density of immune, tumor, and normal cells for $r_{1}=0.27, M(0)=0.25, H(0)=0.01$, and $R(0)=0.9$ when (a) $\tau=5$ (red); (b) $\tau=15$ (green); and (c) $\tau=25$ (blue)

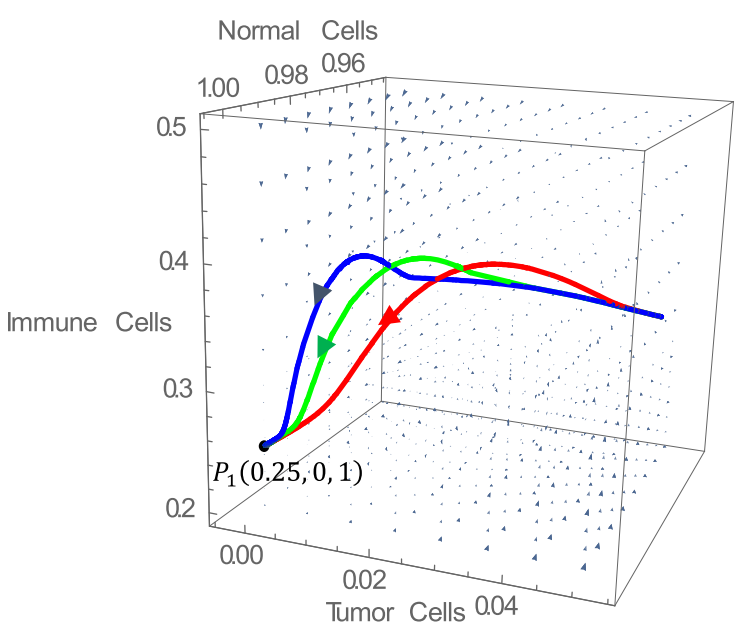

Figure 2 The vector field plot of immune-tumor-normal cells for $r_{1}=0.27, M(0)=0.2, H(0)=0.05$, and $R(0)=0.9$ when (a) $\tau=5$ (red); (b) $\tau=15$ (green); and (c) $\tau=25$ (blue)

nal state when all the tumor cells get eliminated. When time lag increases, the immune system will take some time to provide the right response after discovering the non-selfcells, so in this process, immune cells require more time to completely eradicate the tumor cells.

After verifying our analytical results numerically for $r_{1}<\left(m_{2} \mu / d_{1}\right)+h_{1}$, we proceed with the numerical verification of the other case.

Case 2: $r_{1}>\frac{m_{2} \mu}{d_{1}}+h_{1}$. 


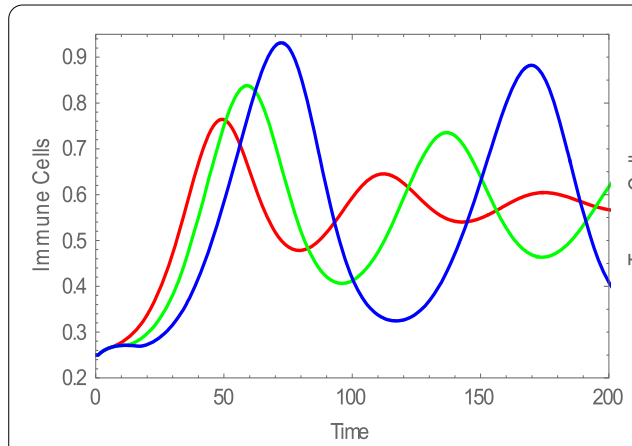

(a)

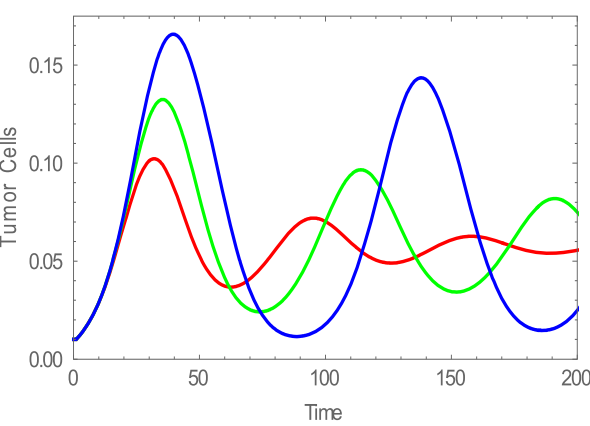

(b)

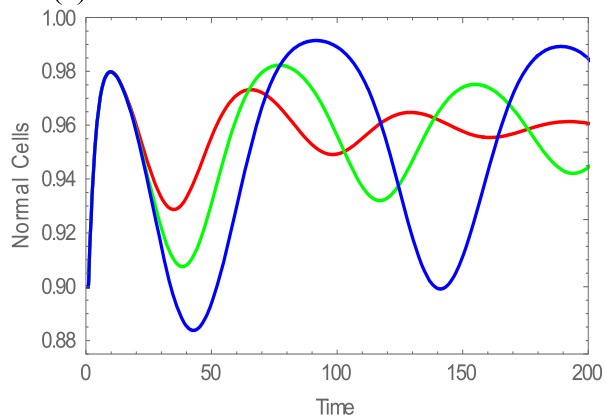

(c)

Figure 3 Density of immune, tumor, and normal cells for $r_{1}=0.4, M(0)=0.25, H(0)=0.01$, and $R(0)=0.9$ when (a) $\tau=5$ (red); (b) $\tau=10$ (green); and (c) $\tau=15$ (blue)

In the case of tumors with a high growth rate $\left(r_{1}=0.4\right)$ (which is in the unstable range), Fig. 3 shows that when the immune system takes a long time to provide an adequate response to the tumor cells for recognition, the tumor growth rate gets faster and the system loses its stability and moves away from the coexisting equilibrium point $P_{2}$.

Therefore, we need to consider the effect of delay in the proliferation of the cells induced by the tumor cells when $r_{1}>\left(m_{2} \mu / d_{1}\right)+h_{1}$. In the event of a long delay, the system will lose stability since other strong factors can occur such as the periodic oscillatory behavior or bifurcation.

\subsection{The coexisting equilibrium point $P_{2}$ and its analysis}

The Jacobian matrix of system (1) at the coexisting equilibrium point $P_{2}\left(M_{2}, H_{2}, R_{2}\right)$ is

$$
J_{P_{2}}=\left(\begin{array}{ccc}
X_{1} e^{-\phi \tau}+X_{2} & X_{5} e^{-\phi \tau}-m_{1} M_{2} & 0 \\
-m_{2} H_{2} & X_{3} & -h_{1} H_{2} \\
0 & -h_{2} R & X_{4}
\end{array}\right),
$$

where

$$
\begin{aligned}
& X_{1}=\frac{\rho H_{2}}{\sigma+H_{2}}, \quad X_{2}=-d_{1}-m_{1} H_{2}, \quad X_{3}=r_{1}-2 r_{1} b H_{2}-m_{2} M_{2}-h_{1} R_{2}, \\
& X_{4}=r_{2}-2 r_{2} R_{2}-h_{2} H_{2}, \quad X_{5}=\frac{\sigma \rho M_{2}}{\left(\sigma+H_{2}\right)^{2}} .
\end{aligned}
$$


The eigenvalues associated with the coexisting equilibrium point $P_{2}\left(M_{2}, H_{2}, R_{2}\right)$ are derived from the characteristic equation

$$
\begin{aligned}
\Omega(\phi, \tau)= & \left(X_{1} e^{-\phi \tau}+X_{2}\right)\left(X_{3} X_{4}-h_{1} h_{2} R_{2} H_{2}\right)+\left(m_{2} H_{2} X_{4} X_{5} e^{-\phi \tau}-m_{1} m_{2} H_{2} M_{2} X_{4}\right) \\
& -\phi\left(\left(X_{3} X_{4}-h_{1} h_{2} R_{2} H_{2}\right)+\left(X_{3}+X_{4}\right)\left(X_{1} e^{-\phi \tau}+X_{2}\right)\right. \\
& \left.+\left(m_{2} H_{2} X_{5} e^{-\phi \tau}-m_{1} m_{2} H_{2} M_{2}\right)\right) \\
& +\phi^{2}\left(\left(X_{3}+X_{4}\right)+\left(X_{1} e^{-\phi \tau}+X_{2}\right)\right)-\phi^{3}=0,
\end{aligned}
$$

or

$$
\Omega(\phi, \tau)=\phi^{3}+X_{11} \phi^{2}+X_{12} \phi+X_{13}+e^{-\tau \phi}\left(Y_{11} \phi^{2}+Y_{12} \phi+Y_{13}\right)=0,
$$

where

$$
\begin{aligned}
& X_{11}=-\left(X_{2}+X_{3}+X_{4}\right) \\
& X_{12}=X_{2} X_{3}+X_{3} X_{4}+X_{2} X_{4}-m_{1} m_{2} M_{2} H_{2}-h_{1} h_{2} R_{2} H_{2} \\
& X_{13}=m_{1} m_{2} M_{2} H_{2} X_{4}+h_{1} h_{2} H_{2} R_{2} X_{2}-X_{2} X_{3} X_{4}, \\
& Y_{11}=-X_{1} \\
& Y_{12}=X_{1} X_{3}+X_{1} X_{4}+m_{2} H_{2} X_{5} \\
& Y_{13}=-\left(m_{2} H_{2} X_{4} X_{5}+X_{1} X_{3} X_{4}-h_{1} h_{2} R_{2} H_{2} X_{1}\right) .
\end{aligned}
$$

For analyzing the effect of delay in our investigation, the following cases are considered: Case (i): $\tau=0$. Without any delay $(\tau=0)$, the characteristic equation reduces to

$$
\phi^{3}+\left(X_{11}+Y_{11}\right) \phi^{2}+\left(X_{12}+Y_{12}\right) \phi+\left(X_{13}+Y_{13}\right)=0
$$

According to the Routh-Hurwitz criterion, all the roots of the equation have negative real parts if

$$
\left(X_{11}+Y_{11}\right)>0, \quad\left(X_{11}+Y_{11}\right)\left(X_{12}+Y_{12}\right)>\left(X_{13}+Y_{13}\right)
$$

where

$$
\begin{aligned}
X_{11}+Y_{11}= & -\left(\frac{\rho H_{2}}{\sigma+H_{2}}-d_{1}-m_{1} H_{2}+r_{1}-2 r_{1} b H_{2}-m_{2} M_{2}\right. \\
& \left.-h_{1} R_{2}+r_{2}-2 r_{2} R_{2}-h_{2} H_{2}\right)
\end{aligned}
$$

and

$$
\begin{aligned}
& \left(X_{11}+Y_{11}\right)\left(X_{12}+Y_{12}\right)-\left(X_{13}+Y_{13}\right) \\
& \quad=\left(d_{1}+m_{1} H_{2}-r_{1}+2 r_{1} b H_{2}+m_{2} M_{2}+h_{1} R_{2}-r_{2}+2 r_{2} R_{2}+h_{2} H_{2}-\frac{\rho H_{2}}{\sigma+H_{2}}\right)
\end{aligned}
$$




$$
\begin{aligned}
& \times\left(\left(\frac{\rho H_{2}}{\sigma+H_{2}}-d_{1}-m_{1} H_{2}+r_{2}-2 r_{2} R_{2}-h_{2} H_{2}\right)\left(r_{1}-2 r_{1} b H_{2}-m_{2} M_{2}-h_{1} R_{2}\right)\right. \\
& +\left(\frac{\rho H_{2}}{\sigma+H_{2}}-d_{1}-m_{1} H_{2}\right)\left(r_{2}-2 r_{2} R_{2}-h_{2} H_{2}\right) \\
& \left.-m_{1} m_{2} M_{2} H_{2}-h_{1} h_{2} R_{2} H_{2}+m_{2} H_{2} \frac{\sigma \rho M_{2}}{\left(\sigma+H_{2}\right)^{2}}\right) \\
& -\left(m_{1} m_{2} M_{2} H_{2}\left(r_{2}-2 r_{2} R_{2}-h_{2} H_{2}\right)+h_{1} h_{2} H_{2} R_{2}\left(d_{1}+m_{1} H_{2}\right)\right. \\
& +\left(\left(d_{1}+m_{1} H_{2}\right)+\frac{\rho H_{2}}{\sigma+H_{2}}\right)\left(r_{1}-2 r_{1} b H_{2}-m_{2} M_{2}-h_{1} R_{2}\right)\left(r_{2}-2 r_{2} R_{2}-h_{2} H_{2}\right) \\
& \left.+\left(m_{2} H_{2}\left(r_{2}-2 r_{2} R_{2}-h_{2} H_{2}\right) \frac{\sigma \rho M_{2}}{\left(\sigma+H_{2}\right)^{2}}+h_{1} h_{2} R_{2} H_{2} \frac{\rho H_{2}}{\sigma+H_{2}}\right)\right) .
\end{aligned}
$$

Thus, if the above conditions are satisfied then the coexisting equilibrium point $P_{2}\left(M_{2}, H_{2}, R_{2}\right)$ will be stable.

Case (ii): $\tau>0$. The immune system needs some time to develop a suitable response by recognizing the tumor cells and, therefore, the time delay is introduced into the model.

The characteristic equation with time delay is

$$
\phi^{3}+X_{11} \phi^{2}+X_{12} \phi+X_{13}+e^{-\tau \phi}\left(Y_{11} \phi^{2}+Y_{12} \phi+Y_{13}\right)=0
$$

The classical Routh-Hurwitz criterion does not apply to the delay system (1), since this equation is transcendental and has an infinite number of solutions. Substituting $\phi=\lambda i$ $(\lambda>0)$ into equation (4) gives

$$
(\lambda i)^{3}+X_{11}(\lambda i)^{2}+X_{12}(\lambda i)+X_{13}+e^{-\tau \lambda i}\left(Y_{11}(\lambda i)^{2}+Y_{12}(\lambda i)+Y_{13}\right)=0
$$

Separation of imaginary and real parts leads to

$$
\begin{aligned}
& -\lambda^{3}+X_{12} \lambda=\left(Y_{13}-\lambda^{2} Y_{11}\right) \sin (\lambda \tau)-\lambda Y_{12} \cos (\lambda \tau), \\
& X_{11} \lambda^{2}-X_{13}=\left(Y_{13}-\lambda^{2} Y_{11}\right) \cos (\lambda \tau)+\lambda Y_{12} \sin (\lambda \tau) .
\end{aligned}
$$

Squaring (5.1) and (5.2) and adding the resulting equations yields

$$
\lambda^{6}+\left(X_{11}^{2}-2 X_{12}-Y_{11}^{2}\right) \lambda^{4}+\left(X_{12}^{2}-2 X_{11} X_{13}+2 Y_{11} Y_{13}-Y_{12}^{2}\right) \lambda^{2}+\left(X_{13}^{2}-Y_{13}^{2}\right)=0
$$

or

$$
\lambda^{6}+p_{11} \lambda^{4}+p_{12} \lambda^{2}+p_{13}=0
$$

Here

$$
\begin{aligned}
p_{11} & =\left(X_{11}^{2}-2 X_{12}-Y_{11}^{2}\right) \\
& =\left(\sigma+H_{2}\right)^{2}\left(-r_{2}+h_{2} H_{2}-r_{1}+m_{2} M_{2}+h_{1} R_{2}-d_{1}-m_{1} H_{2}\right)^{2} \\
& >2\left(\sigma+H_{2}\right)^{2}\left(\left(-r_{2}+h_{2} H_{2}\right)\left(-r_{1}+m_{2} M_{2}+h_{1} R_{2}-d_{1}-m_{1} H_{2}\right)\right.
\end{aligned}
$$




$$
\begin{aligned}
& \left.-\left(-r_{1}+m_{2} M_{2}+h_{1} R_{2}\right)\left(d_{1}+m_{1} H_{2}\right)-m_{1} m_{2} M_{2} H_{2}-h_{1} h_{2} R_{2} H_{2}\right)+\left(\rho H_{2}\right)^{2}, \\
p_{13}= & \left(X_{13}^{2}-Y_{13}^{2}\right) \\
= & \left(\left(-r_{2}+h_{2} H_{2}\right)\left(-r_{1}+m_{2} M_{2}+h_{1} R_{2}\right)\left(d_{1}+m_{1} H_{2}\right)+h_{1} h_{2} R_{2} H_{2}\left(d_{1}+m_{1} H_{2}\right)\right. \\
& \left.+\left(-r_{2}+h_{2} H_{2}\right) m_{1} m_{2} M_{2} H_{2}\right)^{2} \\
& -\left(\left(-r_{2}+h_{2} H_{2}\right) m_{2} H_{2} \frac{\sigma \rho M_{2}}{\left(\sigma+H_{2}\right)^{2}}+\left(-r_{2}+h_{2} H_{2}\right)\left(-r_{1}+m_{2} M_{2}+h_{1} R_{2}\right) \frac{\rho H_{2}}{\sigma+H_{2}}\right. \\
& \left.-h_{1} h_{2} R_{2} H_{2} \frac{\rho H_{2}}{\sigma+H_{2}}\right)^{2} .
\end{aligned}
$$

Using the parameter values listed in Table 1 in equations (7) and (8), we find $p_{11}>0$ and $p_{13}<0$. Now, we can conclude that equation (6) has at least one nonnegative real root and so, the characteristic equation (4) has purely imaginary roots $\pm \lambda_{0} i$ (say). This implies that there is a stability switch at $P_{2}$ as $\tau$ changes.

Eliminating $\sin (\lambda \tau)$ from (5.1) and (5.2) (substituting (5.2) into (5.1)), we get

$$
\begin{gathered}
-\lambda^{3}+X_{12} \lambda=\left(Y_{13}-\lambda^{2} Y_{11}\right)\left(\frac{X_{11} \lambda^{2}-X_{13}-\left(Y_{13}-\lambda^{2} Y_{11}\right) \cos (\lambda \tau)}{\lambda Y_{12}}\right)-\lambda Y_{12} \cos (\lambda \tau) \\
\Rightarrow \quad \lambda Y_{12}\left(-\lambda^{3}+X_{12} \lambda\right) \\
=\left(X_{11} \lambda^{2}-X_{13}\right)\left(Y_{13}-\lambda^{2} Y_{11}\right)-\left(Y_{13}-\lambda^{2} Y_{11}\right)^{2} \cos (\lambda \tau)-\lambda^{2} Y_{12}^{2} \cos (\lambda \tau) \\
\Rightarrow \quad \cos (\lambda \tau)=\frac{\left(Y_{13}-\lambda^{2} Y_{11}\right)\left(X_{11} \lambda^{2}-X_{13}\right)-\lambda Y_{12}\left(-\lambda^{3}+\lambda X_{12}\right)}{\left(Y_{13}-\lambda^{2} Y_{11}\right)^{2}+\lambda^{2} Y_{12}^{2}} .
\end{gathered}
$$

From the above equation, the time lag $\tau_{n}^{*}$ corresponding to $\lambda_{0}$ is given by

$$
\tau_{n}^{*}=\frac{2 n \pi}{\lambda_{0}}+\frac{1}{\lambda_{0}} \arccos \left[\frac{\left(Y_{13}-\lambda_{0}^{2} Y_{11}\right)\left(X_{11} \lambda_{0}^{2}-X_{13}\right)-\lambda_{0} Y_{12}\left(-\lambda_{0}^{3}+X_{12} \lambda_{0}\right)}{\left(Y_{13}-\lambda_{0}^{2} Y_{11}\right)^{2}+\lambda_{0}^{2} Y_{12}^{2}}\right],
$$

where $n$ is an integer.

So, the equilibrium point $P_{2}$ is locally asymptotically stable for all $\left[0, \tau_{0}\right)$ where $\tau_{0}=\tau_{0}^{*}$ (by putting $n=0$ in the above expression of $\tau_{n}^{*}$ ) if conditions $p_{11}>0$ and $p_{13}<0$ (from equations (7) and (8)) are satisfied [33].

\subsection{Occurrence of Hopf bifurcation}

We now investigate the occurrence of a Hopf bifurcation when the delay term $\tau$ passes through the critical value $\tau=\tau_{0}$.

The characteristic equation (4) has purely imaginary eigenvalues $\phi_{0}=i \lambda_{0}$ and $\bar{\phi}_{0}=-i \lambda_{0}$ at $\tau=\tau_{0}$. From equation (4), we have

$$
\Omega\left(\phi_{0}, \tau_{0}\right)=0
$$

and

$$
\begin{aligned}
& \frac{\partial \Omega\left(\phi_{0}, \tau_{0}\right)}{\partial \phi} \\
& \quad=-3 \lambda_{0}^{2}+2 i X_{11} \lambda_{0}+X_{12}+e^{-i \tau_{0} \lambda_{0}}\left(2 i Y_{11} \lambda_{0}+Y_{12}-\tau_{0}\left(-Y_{11} \lambda_{0}^{2}+i Y_{12} \lambda_{0}+Y_{13}\right)\right) \neq 0 .
\end{aligned}
$$


Thus, according to the implicit function theorem, there exists a complex function $\phi=\phi(\tau)$ defined in a neighborhood of $\tau_{0}$ such that $\phi\left(\tau_{0}\right)=\phi_{0}$ and $\Omega(\phi(\tau), \tau)=0$ and

$$
\begin{aligned}
& \frac{d \phi}{d \tau}=-\frac{\frac{\partial \Omega(\phi(\tau), \tau)}{\partial \tau}}{\frac{\partial \Omega(\phi(\tau), \tau)}{\partial \phi}}, \quad \text { for } \tau \text { in a neighborhood of } \tau_{0}, \\
& \frac{d \phi}{d \tau}=\frac{\phi e^{-\tau \phi}\left(Y_{11} \phi^{2}+Y_{12} \phi+Y_{13}\right)}{3 \phi^{2}+2 X_{11} \phi+X_{12}+e^{-\tau \phi}\left(2 Y_{11} \phi+Y_{12}-\tau\left(Y_{11} \phi^{2}+Y_{12} \phi+Y_{13}\right)\right)} .
\end{aligned}
$$

From equations (4) and (9), we obtain the following expression:

$$
\left(\frac{d \phi}{d \tau}\right)^{-1}=\frac{-\left(3 \phi^{2}+2 X_{11} \phi+X_{12}\right)}{\phi\left(\phi^{3}+X_{11} \phi^{2}+X_{12} \phi+X_{13}\right)}+\frac{2 Y_{11} \phi+Y_{12}}{\phi\left(Y_{11} \phi^{2}+Y_{12} \phi+Y_{13}\right)}-\frac{\tau}{\phi} .
$$

Let $\phi(\tau)=\operatorname{Re}(\phi(\tau))+i \operatorname{Im}(\phi(\tau))$. Then, from (10), we find

$$
\begin{aligned}
& \left(\frac{d \operatorname{Re}(\phi(\tau))}{d \tau}\right)_{\tau=\tau_{0}}^{-1} \\
& \quad=\left[\frac{3 \lambda_{0}^{4}-2 \lambda_{0}^{2}\left(2 X_{12}-X_{11}^{2}\right)+X_{12}^{2}-2 X_{11} X_{13}}{\left(X_{13}-X_{11} \lambda_{0}^{2}\right)^{2}+\left(\lambda_{0}^{3}-X_{12} \lambda_{0}\right)^{2}}-\frac{2 \lambda_{0}^{2} Y_{11}^{2}+\left(Y_{12}^{2}-2 Y_{11} Y_{13}\right)}{\left(\lambda_{0} Y_{11}-Y_{13}\right)^{2}+\lambda_{0}^{2} Y_{12}^{2}}\right] \neq 0,
\end{aligned}
$$

which verifies the transversality condition for the Hopf bifurcation. Therefore, system (1) undergoes a Hopf bifurcation at $\tau=\tau_{0}$ and an isolated periodic orbit emerges about $P_{2}$.

Thus, the coexisting equilibrium point $P_{2}$ is locally asymptotically stable for all $\tau=0$. Further, if $X_{11}^{2}-2 X_{12}-Y_{11}^{2}>0$ and $X_{13}^{2}-Y_{13}^{2}<0$, then there exists a positive number $\tau_{0}$ such that $P_{2}$ is locally asymptotically stable for $0<\tau<\tau_{0}$ and unstable for $\tau>\tau_{0}$. Additionally, system (1) undergoes a Hopf bifurcation at $P_{2}$ when $\tau=\tau_{0}$.

\subsection{Verification of existence of limit cycle through simulation}

In this subsection, we verify the above analytical results with numerical simulations for the parameter values listed in Table 1 . The singular point $P_{2}$ is located at $(0.579465,0.0572258$, 0.959124 ), and we have $p_{11}=0.15238135, p_{12}=0.00642814197$, and $p_{13}=-0.0000266$. There exists a single pure real root $\lambda_{0}=0.06156$ for which we found that $\tau_{0}=18.91866$.

Figure 4(a) shows that the equilibrium point $P_{2}$ remains a stable spiral for $\tau<\tau_{0}=$ 18.9186. Physically, it means that the growth of the cancerous tumor is slowed down for a while but not eradicated. From Fig. 4(b), it is seen that a limit cycle, i.e., an isolated periodic solution, appears when the value of $\tau$ is increased to $\tau_{0}=18.91866$. Physically, it means that with the delay of time, tumorous cells begin to show a dominant nature and, therefore, the immune response is insufficient to reduce the rapid growth of the number of tumor cells. After this point, administration of the drug is approved; otherwise, the tumorous growth becomes faster at the expense of immune and normal cells and can eventually lead the patient to a fatal condition.

\subsection{Stability of limit cycle: length of time lag estimation}

In this section, we investigate the stability of bifurcating periodic solutions and estimate the length of time lag preserving the stability of the period-1 limit cycle. For this, system (1) is first linearized around the interior equilibrium point $P_{2}\left(M_{2}, H_{2}, R_{2}\right)$ which gives

$$
\frac{d M}{d t}=\frac{\sigma \rho M^{*} H(t-\tau)}{(\sigma+H(t-\tau))^{2}}-m_{1} M^{*} H+\frac{\rho H^{*} M(t-\tau)}{\sigma+H^{*}}-m_{1} H^{*} M-d_{1} M
$$




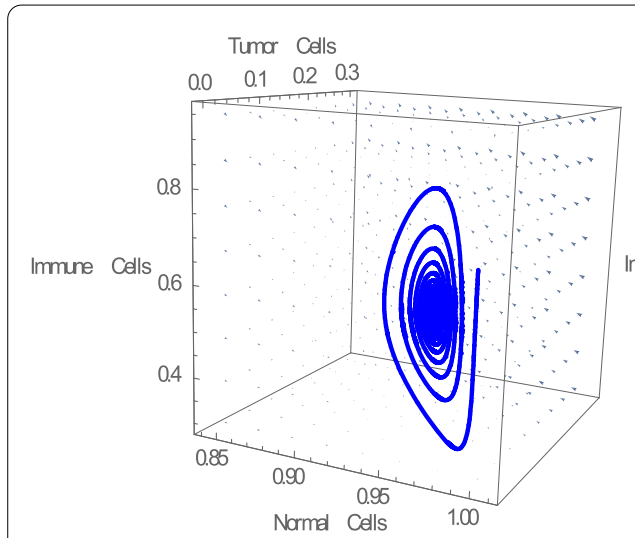

(a)

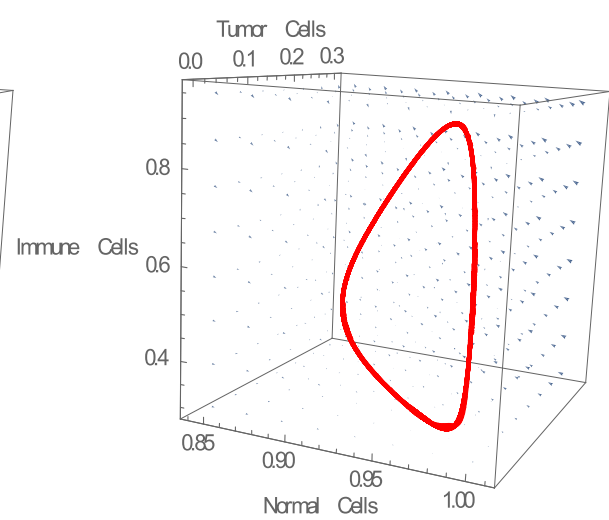

(b)

Figure 4 The phase portrait for the cell populations with $M(0)=0.7, H(0)=0.01$, and $R(0)=0.993$ in the case (a) $\tau<\tau_{0}$, where the steady-state is asymptotically stable and (b) $\tau=\tau_{0}$, where a limit cycle is born around the steady-state with $r_{1}=0.4$

$$
\begin{aligned}
& \frac{d H}{d t}=-m_{2} H^{*} M+\left(r_{1}-2 r_{1} b H^{*}-m_{2} M^{*}-h_{1} R^{*}\right) H-h_{1} H^{*} R, \\
& \frac{d R}{d t}=-h_{2} R^{*} H+\left(r_{2}-2 r_{2} R^{*}-h_{2} H^{*}\right) R .
\end{aligned}
$$

Applying the Laplace transformation to system (11) leads to

$$
\begin{aligned}
& \phi L_{M}(\phi)-\bar{M}(0) \\
& =-d_{1} L_{M}(\phi)-m_{1} H^{*} L_{M}(\phi)-m_{1} M^{*} L_{H}(\phi)+\frac{\sigma \rho M^{*}}{(\sigma+H)^{2}} e^{-\phi \tau} L_{H}(\phi) \\
& \quad+\frac{\rho H^{*}}{\sigma+H^{*}} e^{-\phi \tau} L_{M}(\phi)+\frac{\sigma \rho M^{*}}{(\sigma+H)^{2}} e^{-\phi \tau} K_{H}(\phi)+\frac{\rho H^{*}}{\sigma+H^{*}} e^{-\phi \tau} K_{M}(\phi), \\
& \phi L_{H}(\phi)-\bar{H}(0) \\
& =-m_{2} H^{*} L_{M}(\phi)+\left(r_{1}-2 r_{1} b H^{*}-m_{2} M^{*}-h_{1} R^{*}\right) L_{H}(\phi)-h_{1} H^{*} L_{R}(\phi), \\
& \phi L_{R}(\phi)-\bar{R}(0)=-h_{2} R^{*} L_{H}(\phi)+\left(r_{2}-2 r_{2} R^{*}-h_{2} H^{*}\right) L_{R}(\phi),
\end{aligned}
$$

where

$$
K_{H}(\phi)=\int_{-\tau}^{0} \mathrm{e}^{-\phi \tau} H(t) d t, \quad K_{M}(\phi)=\int_{-\tau}^{0} \mathrm{e}^{-\phi \tau} M(t) d t,
$$

and $L_{M}(\phi), L_{H}(\phi)$, and $L_{R}(\phi)$ are the Laplace transformations of $M(t), H(t)$, and $R(t)$, respectively.

Now, using the theory provided by the classical Nyquist criteria [15] and Freedman et al. [33], the equilibrium point $P_{2}$ will be asymptotically stable if for the expression

$$
P(\phi)=\phi^{3}+X_{11} \phi^{2}+X_{12} \phi+X_{13}+e^{-\tau \phi}\left(Y_{11} \phi^{2}+Y_{12} \phi+Y_{13}\right)
$$


the following conditions hold:

$$
\begin{aligned}
& \operatorname{Re}\left(P\left(i \zeta_{0}\right)\right)=0, \\
& \operatorname{Im}\left(P\left(i \zeta_{0}\right)\right)=0,
\end{aligned}
$$

where $\zeta_{0}$ is the minimal nonnegative root of (12) and (13).

From (12), we have

$$
X_{11} \zeta_{0}^{2}=X_{13}+\left(Y_{13}-\zeta_{0}^{2} Y_{11}\right) \cos \left(\zeta_{0} \tau\right)+\zeta_{0} Y_{12} \sin \left(\zeta_{0} \tau\right)
$$

Using the inequalities $\left|\cos \left(\zeta_{0} \tau\right)\right| \leq 1$ and $\left|\sin \left(\zeta_{0} \tau\right)\right| \leq 1$, we get

$$
\left|X_{11}\right| \zeta_{0}^{2}=\left|X_{13}\right|+\left|Y_{13}\right|+\zeta_{0}^{2}\left|Y_{11}\right|+\zeta_{0}\left|Y_{12}\right|
$$

From (15), we find

$$
\zeta_{+} \leq \frac{\left|Y_{11}\right|+\sqrt{Y_{11}^{2}+4\left(\left|X_{11}\right|-\left|Y_{11}\right|\right)\left(\left|X_{13}\right|+\left|Y_{13}\right|\right)}}{2\left(\left|X_{11}\right|-\left|Y_{11}\right|\right)} .
$$

Also, from (13), we have

$$
\zeta_{0}^{2}<X_{12}-\frac{Y_{13} \sin \left(\zeta_{0} \tau\right)}{\zeta_{0}}+Y_{12} \cos \left(\zeta_{0} \tau\right)+\zeta_{0} Y_{11} \sin \left(\zeta_{0} \tau\right)
$$

Using (14) and (16) gives

$$
\begin{aligned}
& \left(Y_{13}-Y_{11} \zeta_{0}^{2}-X_{11} Y_{12}\right)\left(\cos \left(\zeta_{0} \tau\right)-1\right)+\left(\left(Y_{12}-X_{11} Y_{11}\right) \zeta_{0}+\frac{X_{11} Y_{13}}{\zeta_{0}}\right) \sin \left(\zeta_{0} \tau\right) \\
& \quad<X_{11} X_{12}-X_{13}+X_{11} Y_{12}-Y_{13}+Y_{11} \zeta_{0}^{2} .
\end{aligned}
$$

Through the inequality (16) for $\tau=0$, (17) becomes

$$
\begin{aligned}
& \left(Y_{13}-Y_{11} \zeta_{0}^{2}-X_{11} Y_{12}\right)\left(\cos \left(\zeta_{0} \tau\right)-1\right)+\left(\left(Y_{12}-X_{11} Y_{11}\right) \zeta_{0}+\frac{X_{11} Y_{13}}{\zeta_{0}}\right) \sin \left(\zeta_{0} \tau\right) \\
& \quad<\left(X_{11}+Y_{11}\right)\left(X_{12}+Y_{12}\right)-\left(X_{13}+Y_{13}\right) .
\end{aligned}
$$

Now, the first and second terms of the left-hand side of (18) can be respectively written as

$$
\begin{aligned}
& \left(Y_{13}-Y_{11} \zeta_{0}^{2}-X_{11} Y_{12}\right)\left(\cos \left(\zeta_{0} \tau\right)-1\right)=2\left(Y_{11} \zeta_{0}^{2}+X_{11} Y_{12}-Y_{13}\right) \sin ^{2}\left(\frac{\zeta_{0} \tau}{2}\right) \\
& \quad \leq \frac{1}{2} \zeta_{+}^{2}\left|\left(Y_{12} \zeta_{+}^{2}+X_{11} Y_{12}-Y_{13}\right)\right| \tau^{2}
\end{aligned}
$$

and

$$
\left(\left(Y_{12}-X_{11} Y_{11}\right) \zeta_{0}+\frac{X_{11} Y_{13}}{\zeta_{0}}\right) \sin \left(\zeta_{0} \tau\right) \leq\left(\left|Y_{12}-X_{11} Y_{11}\right| \zeta_{+}^{2}+\left|X_{11}\right|\left|Y_{13}\right|\right) \tau
$$


Therefore, from (18), we find

$$
\chi_{1} \tau^{2}+\chi_{2} \tau \leq \chi_{3}
$$

where

$$
\begin{aligned}
& \chi_{1}=\frac{1}{2} \zeta_{+}^{2}\left|\left(Y_{12} \zeta_{+}^{2}+X_{11} Y_{12}-Y_{13}\right)\right|, \\
& \chi_{2}=\left|Y_{12}-X_{11} Y_{11}\right| \zeta_{+}^{2}+\left|X_{11}\right|\left|Y_{13}\right|, \\
& \chi_{3}=\left(X_{11}+Y_{11}\right)\left(X_{12}+Y_{12}\right)-\left(X_{13}+Y_{13}\right) .
\end{aligned}
$$

Now, expression (18) gives

$$
\tau_{+}=\frac{1}{2 \chi_{1}}\left(-\chi_{2}+\sqrt{\chi_{2}^{2}+4 \chi_{1} \chi_{3}}\right) \text { for } 0 \leq \tau \leq \tau_{+} .
$$

Therefore, the period-1 limit cycle arising in the system preserves stability around the equilibrium $P_{2}$ for the maximum length of the time lag $\tau_{+}$.

\section{Other simulation results}

In this section, we demonstrate a series of numerical simulations associated with the theoretical results obtained in the previous sections. In particular, we are interested in the scenarios where the induced time delay changes the stability, i.e., situations where, for the same set of parameters, a stable steady state becomes unstable with a change in the delays or vice versa. The parameter values have been selected from Table 1 .

Case 1: $r_{1}<\frac{m_{2} \mu}{d_{1}}+h_{1}$. It has already been shown in the figures of Sect. 5.3.

Case 2: $r_{1} \geq \frac{m_{2} \mu}{d_{1}}+h_{1}$.

From Figs. 5 and 6, it is seen that when discrete-time delay $\tau$ increases, the growth of tumor cells also increases, and the system loses its stability and moves away from the coexisting equilibrium point $P_{2}$. When the time $\operatorname{lag} \tau<\tau_{0}$, the equilibrium point $P_{2}$ is asymptotically stable and the solutions converge to a coexisting equilibrium point. The solutions of the system for $\tau<\tau_{0}$ can be seen in Figs. 5(a) and 6(a) where the system has damped oscillations and the coexisting equilibrium point is locally asymptotically stable. Figures 5(b) and 6 (b) show a stable limit cycle with time delay $\tau_{0}=18.91866$, i.e., high competition among the three cell populations, namely tumor, normal, and immune cells.

Furthermore, from Figs. 5(c) and 6(c), it is observed that when the time lag $\tau>\tau_{0}$, the system is unstable. Determination of the bifurcation point is important for the control of tumor cell populations. After the bifurcation point, system (1) will exhibit unstable behavior, which in turn leads to the uncontrolled growth of the tumor (Fig. 5(c)). It was found that immune and normal cells fought against the tumor cells but failed to stop the growth of the tumor cells.

In Figs. 5 and 6, it can be observed that the proliferation of tumor cells depends on the time taken by the immune system's response to the tumor cells. To balance the effect of delay, it is necessary to increase the growth rate of immune cells and the strength of normal cells in their competition against tumor cells. Hence, in the biological sense, the results have practical significance in terms of determining the amount of drugs required to eliminate the tumor, otherwise, the tumor may grow in a periodic way putting the health of the patient in danger. 


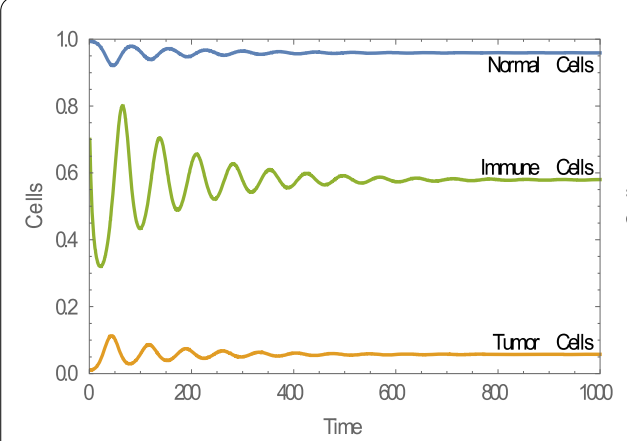

(a)

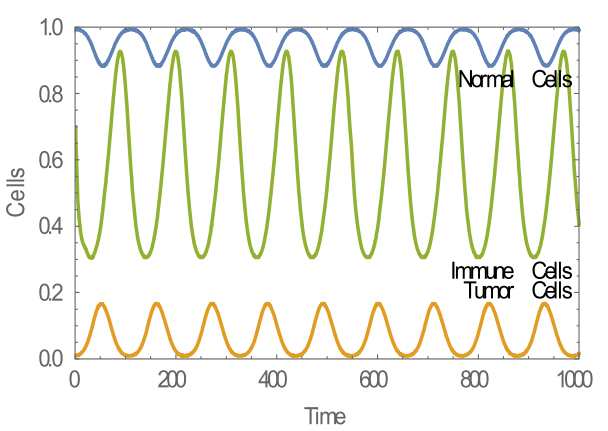

(b)

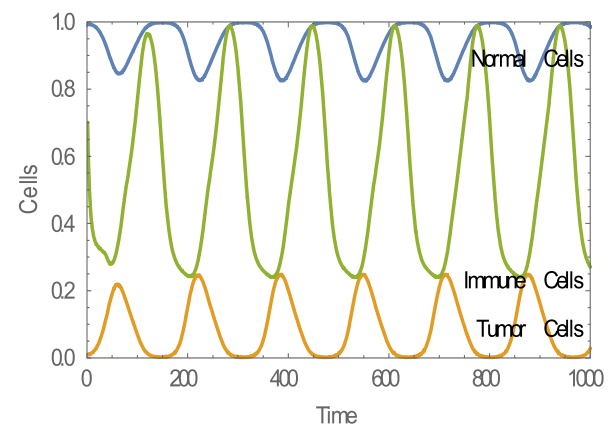

(c)

Figure 5 Density of immune, tumor, and normal cells for $r_{1}=0.4$ and $M(0)=0.7, H(0)=0.01$, and $R(0)=0.993$ when (a) $\tau=8$, (b) $\tau=\tau_{0}=18.91866$, and (c) $\tau=35$

\section{Conclusion}

In this paper, we studied the dynamical behavior of a nonlinear model which was originally proposed by De Pillis et al. by inducing a delay term in the immune system-tumor interaction term. It was done to make the model more realistic. Mathematical analysis of the model demonstrated that, when $r_{1}<\left(m_{2} \mu / d_{1}\right)+h_{1}$, the tumor cells can be eradicated by the combined effort of the immune system and normal cells without the application of any drug. When $r_{1}>\left(m_{2} \mu / d_{1}\right)+h_{1}$ and the immune system takes a long time to recognize the tumor cells to give an adequate response (i.e., delay term is large), the tumor growth rate is faster and the system loses its stability and moves away from the tumor-free equilibrium point $P_{1}$ and, as a result, the immune-normal cells fail to eradicate the tumor load.

To understand more details about the dynamical behavior of the system, we explored the coexisting equilibrium point when $r_{1} \geq\left(m_{2} \mu / d_{1}\right)+h_{1}$. After analysis, it was concluded that the Hopf bifurcation occurs when the time delay increases to $\tau=\tau_{0}$. When the delay term is $\tau<\tau_{0}$, the coexisting equilibrium is locally asymptotically stable at the coexisting equilibrium point $P_{2}$ and becomes unstable when $\tau>\tau_{0}$. In such a situation, to balance the effect of delay, it is necessary to increase the growth rate of immune and normal cells in their competition against tumor cells by employing some drugs.

All of our conclusions are based on the theoretical investigation and some numerical simulations supporting the results. Experimental or clinical verifications will give proper feedback whether the assumptions considered in the formulation of the model and the parameter values used are correct or if they need some modifications. 


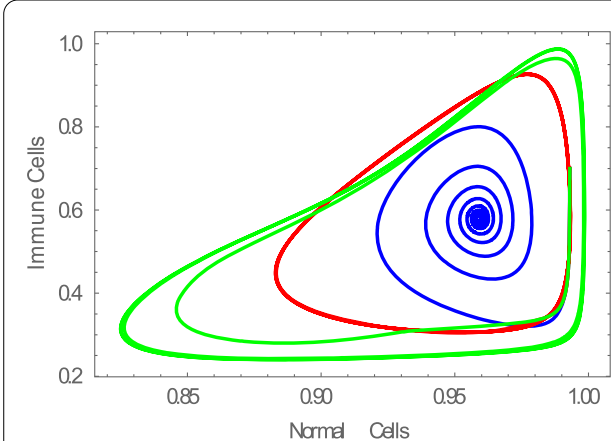

(a)

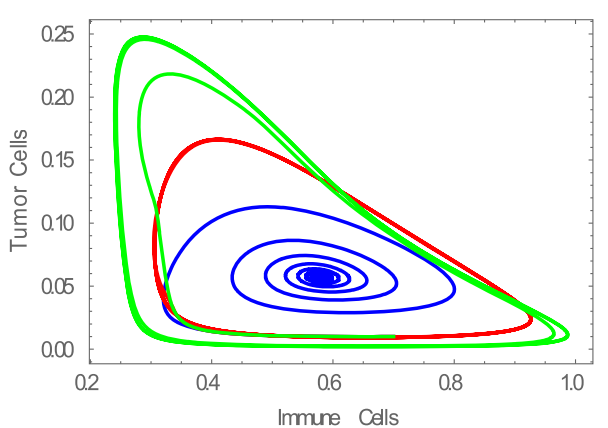

(b)

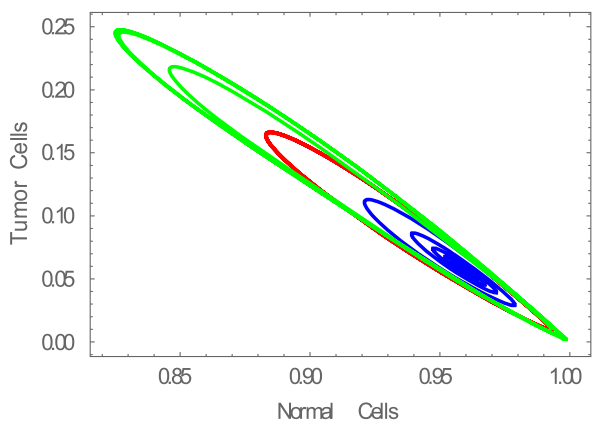

(c)

Figure 6 Parametric plot of immune and tumor cells for $r_{1}=0.4$ and $M(0)=0.7, H(0)=0.01$, and $R(0)=0.993$ when (a) $\tau=8$ (blue); (b) $\tau=\tau_{0}=18.91866$ (red); and (c) $\tau=35$ (green)

Moreover, the numerical simulations involved in the paper were carried out by using the inbuilt software of Mathematica. The order of convergence of the methods employed, the CPU time required, etc., were not addressed as we were interested in studying only the dynamical behaviors shown by the system. Readers who are interested in those aspects and also in different methodologies to carry out the investigation of different problems in epidemiology are referred to the papers [34-43].

\section{Acknowledgements}

Not applicable.

\section{Funding}

There is no funding for this work.

Availability of data and materials

Not applicable.

\section{Declarations}

\section{Competing interests}

The authors declare that they have no competing interests.

Authors' contributions

All authors jointly worked on the results and they read and approved the final manuscript.

\section{Author details}

'Department of Mathematics, Gauhati University, Guwahati 781014, Assam, India. ²Department of Mathematics, Rasht Branch, Islamic Azad University, Rasht, Iran. ${ }^{3}$ Faculty of Engineering and Natural Sciences, Bahcesehir University, Istanbul, Turkey. 


\section{Publisher's Note}

Springer Nature remains neutral with regard to jurisdictional claims in published maps and institutional affiliations.

\section{Received: 5 August 2021 Accepted: 21 January 2022 Published online: 08 February 2022}

\section{References}

1. De Pillis, L.G., Radunskaya, A.E.: The dynamics of optimally controlled tumour model, a case study. Math. Comput. Model. 37, 1221-1244 (2003)

2. Unni, P., Seshaiyer, P.: Mathematical modeling, analysis, and simulation of tumor dynamics with drug interventions. Comput. Math. Methods Med. 2019, 4079298 (2019)

3. Abta, A., Laarabi, H., Alaoui, H.T.: The Hopf bifurcation analysis and optimal control of a delayed SIR epidemic model. Int. J. Anal. 2014, 940819 (2014)

4. Rezapour, S., Mohammadi, H., Samei, M.E.: SEIR epidemic model for COVID-19 transmission by Caputo derivative of fractional order. Adv. Differ. Equ. 2020(490), 1 (2020)

5. Mohammadi, H., Kumar, S., Rezapour, S., Etemad, S.: A theoretical study of the Caputo-Fabrizio fractional modeling for hearing loss due to Mumps virus with optimal control. Chaos Solitons Fractals 144, 110668 (2021)

6. Rezapour, S., Mohammadi, H.: A study on the AH1N1/09 influenza transmission model with the fractional Caputo-Fabrizio derivative. Adv. Differ. Equ. 2020(488), 1 (2020)

7. Aydogan, S.M., Baleanu, D., Mohammadi, H., Rezapour, S.: On the mathematical model of Rabies by using the fractional Caputo-Fabrizio derivative. Adv. Differ. Equ. 2020(382), 1 (2020)

8. De Pillis, L.G., Fister, K.R., Gu, W., Collins, C., Daub, M., Gross, D., Moore, J., Preskill, B.: Mathematical model creation for cancer chemo-immunotherapy. Comput. Math. Methods Med. 10, 165-184 (2009)

9. De Pillis, L.G., Radunskaya, A.E.: A mathematical tumour model with immune resistance and drug therapy: an optimal control approach. J. Theor. Med. 3, 79-100 (2001)

10. Kartal, S.: Mathematical modeling and analysis of tumor-immune system interaction by using Lotka-Volterra predator-prey-like model with piecewise constant arguments. Period. Eng. Nat. Sci. 2, 7-12 (2014)

11. Pang, L., Liu, S., Zhang, X., Tian, T.: Mathematical modeling and dynamic analysis of anti-tumor immune response. J. Appl. Math. Comput. 62, 473-488 (2019)

12. Sardar, M., Biswas, S., Khajanchi, S.: The impact of distributed time delay in a tumor-immune interaction system. Chaos Solitons Fractals 142, 110483 (2021)

13. Galach, M.: Dynamics of the tumour-immune system competition: the effect of time delay. Int. J. Appl. Math. Comput. Sci. 13, 395-406 (2003)

14. McCluskey, C.C.: Complete global stability for an SIR epidemic model with delay-distributed or discrete. Nonlinear Anal., Real World Appl. 11, 55-59 (2010)

15. Banerjee, S.: Immunotherapy with interleukin-2: a study based on mathematical modelling. Int. J. Appl. Math. Comput. Sci. 18, 389-398 (2008)

16. Li, H., Takeuchi, Y: Dynamics of the density dependent predator-prey system with Beddington-DeAngelis functional response. J. Math. Anal. Appl. 374, 644-654 (2011)

17. Yu, P.: Closed-form conditions of bifurcation points for general differential equations. Int. J. Bifurc. Chaos 15 , 1467-1483 (2005)

18. Ghosh, D., Khajanchi, S., Mangiarotti, S., Denis, F., Dana, S.K., Letellier, C.: How tumour growth can be delayed interactions between cancer cells and the microenviroment? Biosystems 158, 17-30 (2017)

19. Zhang, J., Jin, Z., Yan, J., Sun, G.: Stability and Hopf bifurcation in a delayed competition system. Nonlinear Anal., Theory Methods Appl. 70, 658-670 (2009)

20. Li, F., Li, H.: Hopf bifurcation of a predator-prey model with time delay and stage structure for the prey. Math. Comput. Model. 55, 672-679 (2012)

21. Al-Mahdi, A.M., Khirallah, M.Q.: Bifurcation analysis of a model of cancer. Eur. Sci. J. 12, 1857-7881 (2016)

22. Khoshnevisan, L., Liu, X., Salmasi, F.R.: Stability and Hopf bifurcation analysis of a TCP/RAQM network with ISMC procedure. Chaos Solitons Fractals 118, 255-273 (2019)

23. Beretta, E., Takeuchi, Y.: Global stability of single-species diffusion Volterra models with continuous time delays. Bull. Math. Biol. 49, 431-448 (1987)

24. Yafia, R.: Stability of limit cycle in a delayed model for tumour immune system competition with negative immune response. Discrete Dyn. Nat. Soc. 2006, 58463 (2006)

25. Xu, R., Ma, Z: Global stability analysis of a delay SEIRS epidemic model with saturation incidence rate. Nonlinear Dyn. $61,229-239(2010)$

26. Rihan, F.A., Abdelrahman, D.H., Al-Maskari, F., Ibrahim, F., Abdeen, M.A.: Delay differential model for tumour-immune response with chemo-immunotherapy and optimal control. Comput. Math. Methods Med. 2014, 982978 (2014)

27. Xu, S., Wei, X., Zhang, F.: A time-delayed mathematical model for tumor growth with the effect of a periodic therapy Comput. Math. Methods Med. 2016, 3643019 (2016)

28. Yua, M., Dong, Y., Takeuchi, Y.: Dual role of delay effects in a tumour-immune system. J. Biol. Dyn. 11, 334-347 (2017)

29. Malinzi, J.: Mathematical analysis of a mathematical model of chemovirotherapy: effect of drug infusion method. Comput. Math. Methods Med. 2019, 7576591 (2019)

30. El Alami Laaroussi, A., El Hia, M., Rachik, M., Ghazzali, R.: Analysis of a multiple delays model for treatment of cancer with oncolytic virotherapy. Comput. Math. Methods Med. 2019, 1732815 (2019)

31. Villasana, M., Radunskaya, A.: A delay differential equation model for tumor growth. Math. Biol. 47, 270-294 (2003)

32. Yang, X., Chen, L., Chen, J.: Permanence and positive periodic solution for the single-species non-autonomous delay diffusive models. Comput. Math. Appl. 32, 109-116 (1996)

33. Freedman, H.I., Erbe, L., Rao, V.S.H.: Three species food chain models with mutual interference and time delays. Math. Biosci. 80, 57-80 (1986)

34. Akram, T., Abbas, M., Riaz, M.B., Ismail, A.I., Ali, N.M.: An efficient numerical technique for solving time fractional Burgers equation. Alex. Eng. J. 59, 2201-2220 (2020)

35. Khalid, N., Abbas, M., Iqbal, M.K., Baleanu, D.: A numerical investigation of Caputo time fractional Allen-Cahn equation using redefined cubic B-spline functions. Adv. Differ. Equ. 2020(158), 1 (2020) 
36. Iqbal, A., Siddiqui, M.J., Muhi, I., Abbas, M., Akram, T.: Nonlinear waves propagation and stability analysis for planar waves at far field using quintic B-spline collocation method. Alex. Eng. J. 59, 2695-2703 (2020)

37. Khalid, N., Abbas, M., lqbal, M.K., Singh, J., Ismail, A.I.M.: A computational approach for solving time fractional differential equation via spline functions. Alex. Eng. J. 59, 3061-3078 (2020)

38. Akram, T., Abbas, M., Iqbal, A., Baleanu, D., Asad, J.H.: Novel numerical approach based on modified extended cubic B-spline functions for solving non-linear time-fractional telegraph equation. Symmetry 12(7), 1154 (2020)

39. Akram, T., Abbas, M., Ali, A., labal, A., Baleanu, D.: A numerical approach of a time fractional reaction-diffusion model with a non-singular kernel. Symmetry 12(1653), 1-19 (2020)

40. Amin, M., Abbas, M., Iqbal, M.K., Baleanu, D.: Numerical treatment of time-fractional Klein-Gordon equation using redefined extended cubic B-spline functions. Front. Phys. 8(288), 1-13 (2020)

41. Amin, M., Abbas, M., Iqbal, M.K., Ismail, A.I.M., Baleanu, D.: A fourth order non-polynomial quintic spline collocation technique for solving time fractional super diffusion equations. Adv. Differ. Equ. 2019(514), 1 (2019)

42. Khalid, N., Abbas, M., Iqbal, M.K.: Non-polynomial quintic spline for solving fourth-order fractional boundary value problems involving product terms. Appl. Math. Comput. 349, 393-407 (2019)

43. Iqbal, M.K., Abbas, M., Wasim, I.: New cubic B-spline approximation for solving third order Emden-Flower type equations. Appl. Math. Comput. 331, 319-333 (2018)

\section{Submit your manuscript to a SpringerOpen ${ }^{\mathcal{O}}$ journal and benefit from:}

- Convenient online submission

- Rigorous peer review

- Open access: articles freely available online

- High visibility within the field

Retaining the copyright to your article 